\title{
Coefficient estimates for some subclasses of bi-univalent functions related to $\mathbf{m}$-fold symmetry
}

\author{
WaggasGalibAtshan ${ }^{1}$ SalwaKalfKazim ${ }^{2}$ \\ Department of Mathematics, College of Computer Science and Information Technology, University of \\ AL-Qadisiyah , Diwaniyah-Iraq \\ E-mail: waggas.galib@qu.edu.iqwaggashnd@gmail.com
}

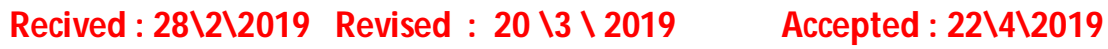

Available online : $2 / 5 / 2019$

\begin{abstract}
:
The purpose of present paper is to introduce and investigate two new subclasses $\mathcal{N}_{\sum \boldsymbol{m}}(\boldsymbol{\tau}, \boldsymbol{\gamma}, \boldsymbol{\alpha})$ and $\mathcal{N}_{\sum \boldsymbol{m}}(\boldsymbol{\tau}, \boldsymbol{\gamma}, \boldsymbol{\beta})$ of analytic and $\mathrm{m}$-fold symmetric bi- univalent functions in the open unit disk. Among other results belonging to these subclasses upper coefficients bounds $\left|\boldsymbol{a}_{\boldsymbol{m + 1}}\right|$ and $\left|\boldsymbol{a}_{\mathbf{2 m + 1}}\right|$ are obtained in this study. Certain special cases are also indicated .
\end{abstract}

Keywords:m-fold symmetry, bi-univalent functions, coefficient estimates.

Mathematics Subject Classification: 30C45. 


\section{Introduction}

Let $S$ denote the family of functions analytic in the open unit $\operatorname{disk} U=\{z: z \in \mathbb{C},|z|<1\}$

and normalized by the conditions $f(0)=f^{\prime}(0)-1=0$ and having the form

$f(z)=z+\sum_{k=2}^{\infty} a_{k} z^{k}$

Also let $\mathcal{A}$ denote the subclass of functions in $S$ which are univalent in $U$.

The Koebe One Quarter Theorem (e.g., see [6]) ensures that the image of $U$ under every

univalent function $f(z) \in S$ contains the disk of radius $1 / 4$ . Thus every univalent function

$f$ has an inverse $f^{-1}$ satisfying

$$
f^{-1}(f(z))=z, \quad(z \in U)
$$

and

$$
f\left(f^{-1}(w)\right)=w \quad, \quad\left(|w|<r \circ(f), r_{\circ}(f) \geq \frac{1}{4}\right)
$$

where

$g(w)=f^{-1}(w)=w-a_{2} w^{2}+\left(2 a_{2}^{2}-a_{3}\right) w^{3}-$ $\left(5 a_{2}^{3}-5 a_{2} a_{3}+a_{4}\right) w^{4}+\cdots$.

A function $f \in S$ is said to be bi-univalent in $U$ if both $f$ and $f^{-1}$ are univalent in $U$.

Let $\sum$ denotes the class of analytic and bi-univalent functions in $U$. Some examples of functions in class $\sum$ are

$$
\begin{aligned}
h_{1}(z)=\frac{z}{1-z}, & h_{2}(z) \\
= & -\log (1-z), \\
& h_{3}(z)=\frac{1}{2} \log \left(\frac{1+z}{1-z}\right), \quad z \in U .
\end{aligned}
$$

For each function $f \in \mathcal{A}$, the function $h(z)=\left(f\left(z^{m}\right)\right)^{\frac{1}{m}}$, $(z \in U, m \in \mathbb{N})$ is univalent and maps the unit disk $U$ into a region with $\mathrm{m}$-fold symmetry . A function is said to be m-fold symmetric (see $[9,10]$ ) if it has the following normalized form :

$f(z)=z+\sum_{k=1}^{\infty} a_{m k+1} z^{m k+1},(z \in U, m \in \mathbb{N})$.

We denote $S_{m}$ the class of m-fold symmetric univalent functions in $U$, which are normalized by the series expansion (3). In fact, the functions in the class $\mathcal{A}$ are one-fold symmetric . Analogous to the concept of $\mathrm{m}$-fold symmetric univalent functions, we here introduced the concept of m-fold symmetric univalent functions, we here introduced the concept of m-fold symmetric bi-univalent functions . Each function $f \in \sum$ generates an m-fold symmetric bi-univalent function for each integer $m \in \mathbb{N}$. Furthermore, for the normalized form of $f$ is given by (3), they obtained the series expansion for $f^{-1}$ as follows :

$g(w)=w-a_{m+1} w^{m+1}+\left[(m+1) a_{m+1}^{2}-a_{2 m+1}\right] w^{2 m+1}-$

$\left[\frac{1}{2}(m+1)(3 m+2) a_{m+1}^{2}-(3 m+2) a_{m+1} a_{2 m+1}+\right.$

$\left.a_{3 m+1}\right] w^{3 m+1}+\cdots,(4)$

where $f^{-1}=g$. We denote by $\sum_{m}$ the class of $\mathrm{m}$-fold symmetric bi-univalent functions in $U$. It is easily seen that for $m=1$, the formula (4) coincides with the formula (2) of the class $\Sigma$. Some examples of $\mathrm{m}$-fold symmetric bi-univalent functions are given as follows :

$\left(\frac{z^{m}}{1-z^{m}}\right)^{\frac{1}{m}},\left[\frac{1}{2} \log \left(\frac{1+z^{m}}{1-z^{m}}\right)\right]^{\frac{1}{m}}$ and

$\left[-\log \left(1-z^{m}\right)\right]^{\frac{1}{m}}$

with the corresponding inverse functions

$\left(\frac{w^{m}}{1+w^{m}}\right)^{\frac{1}{m}},\left(\frac{e^{2 w^{m}}-1}{e^{2 w^{m}}+1}\right)^{\frac{1}{m}}$ and $\left(\frac{e^{w^{m}}-1}{e^{w^{m}}}\right)^{\frac{1}{m}}$

respectively .

Recently , many authors investigated bounds for various subclass of $\mathrm{m}$-fold bi-univalent functions (see $[1,2,3,4,5,7,9,12,13,15])$.The aim of the present paper is to introduce the new subclass $\mathcal{N}_{\sum_{m}}(\tau, \gamma ; \alpha)$ and $\mathcal{N}_{\Sigma m}(\tau, \gamma ; \beta)$ of $\sum_{m}$ and find estimates on the coefficients $\left|a_{m+1}\right|$ and $\left|a_{2 m+1}\right|$ for functions in each of these new subclass.

In order to prove our main results, we require the following lemma .

Lemma 1.([6]).If $h \in \mathcal{P}$, then $\left|c_{k}\right| \leq 2$ for each $k \in \mathbb{N}$, where $\mathcal{P}$ is the family of all functions $h$ analytic in $U$ for which

$\operatorname{Re}(h(z))>0 \quad, \quad(z \in U)$

where

$h(z)=1+c_{1} z+c_{2} z^{2}+\cdots . \quad(z \in U)$

Definition 1. A function $f(z) \in \sum_{m}$ given by (3) is said to be in the class $\mathcal{N}_{\Sigma m}(\tau, \gamma ; \alpha)$ if the following condition are satisfied :

$\left|\arg \left(1+\frac{1}{\tau}\left[\frac{(1+\gamma) z^{2} f^{\prime \prime}(z)+z f^{\prime}(z)}{(1+\gamma) z f^{\prime}(z)-\gamma f(z)}-1\right]\right)\right|<\frac{\alpha \pi}{2}$

$(z \in U)$

and 
$\left|\arg \left(1+\frac{1}{\tau}\left[\frac{(1+\gamma) w^{2} g^{\prime \prime}(w)+w g^{\prime}(w)}{(1+\gamma) w g^{\prime}(w)-\gamma g(w)}-1\right]\right)\right|<\frac{\alpha \pi}{2}$

$(w \in U)(6)$

$(0<\alpha \leq 1 ; \tau \in \mathbb{C} \backslash\{0\} ; 0 \leq \gamma<1)$,

where the function $g=f^{-1}$ is given by (4).

Definition 2. A function $f(z) \in \sum_{m}$ given by (3) is said to be in the class $\mathcal{N}_{\Sigma_{m}}(\tau, \gamma ; \beta)$ if the following conditions are satisfied :

$R e\left(1+\frac{1}{\tau}\left[\frac{(1+\gamma) z^{2} f^{\prime \prime}(z)+z f^{\prime}(z)}{(1+\gamma) z f^{\prime}(z)-\gamma f(z)}-1\right]\right)>\beta$,

$(z \in U)$

and

$\operatorname{Re}\left(1+\frac{1}{\tau}\left[\frac{(1+\gamma) \mathrm{w}^{2} \mathrm{~g}^{\prime \prime}(\mathrm{w})+\mathrm{wg}^{\prime}(\mathrm{w})}{(1+\gamma) \mathrm{wg}^{\prime}(\mathrm{w})-\gamma \mathrm{g}(\mathrm{w})}-1\right]\right)>\beta$,

$(w \in U)(8)$

$(0 \leq \beta<1 ; \tau \in \mathbb{C} \backslash\{0\} ; 0 \leq \gamma<1)$,

where the function $g=f^{-1}$ is given by (4) .

\section{Coefficient Estimates for the Functions Class $\mathcal{N}_{\sum m}(\tau, \gamma ; \boldsymbol{\alpha})$}

We begin this section by finding the estimates on the coefficients $\left|a_{m+1}\right|$ and $\left|a_{2 m+1}\right|$ for functions in the class $\mathcal{N}_{\Sigma_{M}}(\tau, \gamma ; \alpha)$.

Theorem 2.1 Let $f(z) \in \mathcal{N}_{\Sigma m}(\tau, \gamma ; \alpha)(0<\alpha \leq 1 ; \tau \in \mathbb{C} \backslash$ $\{0\}, 0 \leq \gamma<1)$ be of the form (3). Then

$\left|a_{m+1}\right| \leq$ $2 \alpha|\tau|$

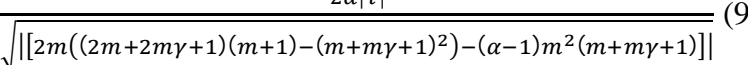

and

$\left|a_{2 m+1}\right| \leq \frac{2 \alpha^{2}|\tau|^{2}(m+1)}{m^{2}(m+m \gamma+1)^{2}}+\frac{\alpha|\tau|}{m(2 m+2 m \gamma+1)}$

Proof. It follows from (5) and (6) that

$1+\frac{1}{\tau}\left[\frac{(1+\gamma) z^{2} f^{\prime \prime}(z)+z f^{\prime}(z)}{(1+\gamma) z f^{\prime}(z)-\gamma f(z)}-1\right]=[p(z)]^{\alpha}$

and

$1+\frac{1}{\tau}\left[\frac{(1+\gamma) w^{2} g^{\prime \prime}(w)+w g^{\prime}(w)}{(1+\gamma) w g^{\prime}(w)-\gamma g(w)}-1\right]=[q(w)]^{\alpha}$,

where the functions $p(z)$ and $q(w)$ are in $\mathcal{P}$ and have the following series representations:

$p(z)=1+p_{m} z^{m}+p_{2 m} z^{2 m}+p_{3 m} z^{3 m}+\cdots$ and

$q(w)=1+q_{m} w^{m}+q_{2 m} w^{2 m}+q_{3 m} w^{3 m}+\cdots$.

Now ,equating the coefficients in (11) and (12),we obtain

$\frac{m(m+m \gamma+1) a_{m+1}}{\tau}=\alpha p_{m}$,

$\frac{\left(2 m(2 m+2 m \gamma+1) a_{2 m+1}-m(m+m \gamma+1)^{2} a_{m+1}^{2}\right)}{\tau}=\alpha p_{2 m}+\frac{\alpha(\alpha-1)}{2} p_{m}^{2}$, (16)

and

$\frac{-m(m+m \gamma+1) a_{m+1}}{\tau}=\alpha q_{m}$ (17) $\frac{\left(2 m(2 m+2 m \gamma+1)\left[(m+1) a_{m+1}^{2}-a_{2 m+1}\right]-m(m+m \gamma+1)^{2} a_{m+1}^{2}\right)}{\tau}=$

$\alpha q_{2 m}+\frac{\alpha(\alpha-1)}{2} q_{m}^{2}$.

From (15)and (17), we find

$p_{m}=-q_{m}(19)$

and

$2 \frac{m^{2}(m+m \gamma+1)^{2} a_{m+1}^{2}}{\tau^{2}}=\alpha^{2}\left(p_{m}^{2}+q_{m}^{2}\right)$

From (16),(18) and (20), we get

$\frac{\left((2 m+2 m \gamma+1)(m+1)-(m+m \gamma+1)^{2}\right) 2 m a_{m+1}^{2}}{\tau}$

$=\alpha\left(p_{2 m}+q_{2 m}\right)+\frac{\alpha(\alpha-1)}{2}\left(p_{m}^{2}+q_{m}^{2}\right)$

$=\alpha\left(p_{2 m}+q_{2 m}\right)+\frac{(\alpha-1) m^{2}(m+m \gamma+1)}{\alpha \tau^{2}} a_{m+1}^{2}$.

Therefore, we have

$a_{m+1}^{2}=$

$\frac{\alpha^{2} \tau^{2}\left(p_{2 m}+q_{2 m}\right)}{\left[2 m\left((2 m+2 m \gamma+1)(m+1)-(m+m \gamma+1)^{2}\right)-(\alpha-1) m^{2}(m+m \gamma+1)\right]}$.

Applying Lemma 1 for the coefficients $p_{2 m}$ and $q_{2 m}$, we have

$\left|a_{m+1}\right| \leq$

$\frac{2 \alpha|t|}{\sqrt{\left|\left[2 m\left((2 m+2 m \gamma+1)(m+1)-(m+m \gamma+1)^{2}\right)-(\alpha-1) m^{2}(m+m \gamma+1)\right]\right|}}$.

This gives the desired bound for $\left|a_{m+1}\right|$ as asserted in (9). In order to find the bound onl $a_{2 m+1} l$, by subtracting (18) from (16), we get

$\frac{2 \mathrm{~m}\left[(2 \mathrm{~m}+2 \mathrm{~m} \gamma+1) \mathrm{a}_{2 \mathrm{~m}+1}-(2 \mathrm{~m}+2 \mathrm{~m} \gamma+1)(\mathrm{m}+1) \mathrm{a}_{\mathrm{m}+1}^{2}\right]}{\tau}=\alpha\left(\mathrm{p}_{2 \mathrm{~m}}\right.$

$\left.\mathrm{q}_{2 \mathrm{~m}}\right)+\frac{\alpha(\alpha-1)}{2}\left(\mathrm{p}_{\mathrm{m}}^{2}-\mathrm{q}_{\mathrm{m}}^{2}\right)$.

It follows from(19) and (24) that

$a_{2 m+1}=\frac{\alpha^{2} \tau^{2}\left(p_{m}^{2}+q_{m}^{2}\right)(m+1)}{4 m(m+m \gamma+1)^{2}}+\frac{\alpha \tau\left(p_{2 m}-q_{2 m}\right)}{4 m(2 m+2 m \gamma+1)}$ 
Applying Lemma 1 once again for the coefficients $p_{m}, p_{2 m}, q_{m}$ and $q_{2 m}$,we readily obtain

$\left|a_{2 m+1}\right| \leq \frac{2 \alpha^{2}|\tau|^{2}(m+1)}{m^{2}(m+m \gamma+1)^{2}}+\frac{\alpha|\tau|}{m(2 m+2 m \gamma+1)}$.

\section{Coefficient Bounds for the Functions Class $\mathcal{N}_{\sum m}(\tau, \gamma ; \boldsymbol{\beta})$}

This section is devoted to find the estimates on the coefficients $\left|a_{2 m+1}\right|$ and $\left|a_{m+1}\right|$ for functions in the class $\mathcal{N}_{\Sigma m}(\tau, \gamma ; \beta)$.

Theorem 3.1 Let $f(z) \in \mathcal{N}_{\sum m}(\tau, \gamma ; \beta)(0 \leq \beta<1 ; \tau \in \mathbb{C} \backslash$ $\{0\}, 0 \leq \gamma<1)$ be of the form (3).

Then

$\left|a_{m+1}\right| \leq \sqrt{\frac{2|\tau|(1-\beta)}{m\left((2 m+2 m \gamma+1)(m+1)-(m+m \gamma+1)^{2}\right)}}$

and

$\left|a_{2 m+1}\right| \leq \frac{4|\tau|^{2}(1-\beta)^{2}(m+1)}{m^{2}(m+m \gamma+1)^{2}}+\frac{2|\tau|(1-\beta)}{m(2 m+2 m \gamma+1)}(28)$

Proof. It follows from (7) and (8) that there exist , $p, q \in \mathcal{P}$ such that

$1+\frac{1}{\tau}\left[\frac{(1+\gamma) z^{2} f^{\prime \prime}(z)+z f^{\prime}(z)}{(1+\gamma) z f^{\prime}(z)-\gamma f(z)}-1\right]=\beta+(1-\beta) p(z)$

and

$1+\frac{1}{\tau}\left[\frac{(1+\gamma) w^{2} g^{\prime \prime}(w)+w g^{\prime}(w)}{(1+\gamma) w g^{\prime}(w)-\gamma g(w)}\right]=\beta+(1-\beta) q(w)$,

where $p(z)$ and $q(z)$ have the forms (13) and (14), respectively. By suitably comparing coefficients in (29) and (30), we get

$$
\begin{aligned}
& \frac{m(m+m \gamma+1) a_{m+1}}{\tau}=(1-\beta) p_{m}, \text {, 31) } \\
& \frac{\left(2 m(2 m+2 m \gamma+1) a_{2 m+1}-m(m+m \gamma+1)^{2} a_{m+1}^{2}\right)}{\tau}=(1-\beta) p_{2 m}, \\
& \frac{-m(m+m \gamma+1) a_{m+1}}{\tau}=(1-\beta) q_{m}, \\
& \frac{\left(2 m(2 m+2 m \gamma+1)\left[(m+1) a_{m+1}^{2}-a_{2 m+1}\right]-m(m+m \gamma+1)^{2} a_{m+1}^{2}\right)}{\tau}= \\
& (1-\beta) q_{2 m} \cdot(34)
\end{aligned}
$$

From (31) and (33), we find

$p_{m}=-q_{m}(35)$

and

$\frac{2 m^{2}(m+m \gamma+1)^{2} a_{m+1}^{2}}{\tau^{2}}=(1-\beta)^{2}\left(p_{m}^{2}+q_{m}^{2}\right)$

Adding (32) and (34), we have $\frac{\left((2 m+2 m \gamma+1)(m+1)-(m+m \gamma+1)^{2}\right) 2 m a_{m+1}^{2}}{\tau}=(1-\beta)\left(p_{2 m}+\right.$ $\left.q_{2 m}\right) \cdot(37)$

Applying Lemma 1, we obtain

$\left|a_{m+1}\right| \leq \sqrt{\frac{2|\tau|(1-\beta)}{m\left((2 m+2 m \gamma+1)(m+1)-(m+m \gamma+1)^{2}\right)}}$.

This is the bound on $\left|a_{m+1}\right|$ asserted in (27).

In order to find the bound on $\left|a_{2 m+1}\right|$, by subtracting (34) form (32), we get

$\frac{2 m\left[(2 m+2 m \gamma+1) a_{2 m+1}-(2 m+2 m \gamma+1)(m+1) a_{m+1}^{2}\right]}{\tau}=(1-\beta)\left(p_{2 m}-\right.$ $q_{2 m)}(38)$

Or ,equivalently,

$a_{2 m+1}=$

$\frac{2 m(2 m+2 m \gamma+1)(m+1) a_{m+1}^{2}}{2 m(2 m+2 m \gamma+1)}+\frac{\tau(1-\beta)\left(p_{2 m}-q_{2 m}\right)}{2 m(2 m+2 m \gamma+1)}(39)$

It follows from (35) and (36) that

$a_{2 m+1}=$

$\frac{\tau^{2}(1-\beta)^{2}(m+1)\left(p_{m}^{2}+q_{m}^{2}\right)}{2 m^{2}(m+m \gamma+1)^{2}}+\frac{\tau(1-\beta)\left(p_{2 m}-q_{2 m}\right)}{2 m(2 m+2 m \gamma+1)}$

Applying lemma 1 once again for the coefficients $p_{m}, p_{2 m}, q_{m}$ and $q_{2 m}$, we easily obtain

$\left|a_{2 m+1}\right| \leq \frac{4|\tau|^{2}(1-\beta)^{2}(m+1)}{m^{2}(m+m \gamma+1)^{2}}+\frac{2|\tau|(1-\beta)}{m(2 m+2 m \gamma+1)}$.

\section{Corollaries and Consequencess}

For one-fold symmetric bi-univalent functions and $\tau=$ 1 , Theorem 2.1 and Theorem 3.1 reduce to Corollary 1 and Corollary 2 , respectively, which were proven very recently by Frasin[8]( see also [11]).

Corollary 4 . Let $f(z) \in \mathcal{N}_{\Sigma}(\alpha, \gamma)(0<\alpha \leq 1 ; 0 \leq \gamma<1)$ be of the form (1).

Then

$\left|a_{2}\right| \leq \frac{2 \alpha}{\sqrt{2(3-\alpha)-\gamma(\gamma+\alpha-1)}}(42)$

and

$\left|a_{3}\right| \leq \frac{4 \alpha^{2}}{(2+\gamma)^{2}}+\frac{\alpha}{(3+2 \gamma)}$.

Corollary 5 . Let $f(z) \in \mathcal{N}_{\Sigma}(\beta, \gamma)(0<\alpha \leq 1 ; 0 \leq \gamma<1)$ be of the form (1).

Then 
$\left|a_{2}\right| \leq \sqrt{\frac{2(1-\beta)}{\left(2+2 \gamma+\gamma^{2}\right)}}$

and

$\left|a_{3}\right| \leq \frac{8(1-\beta)^{2}}{(2+\gamma)^{2}}+\frac{2(1-\beta)}{(3+2 \gamma)}(45)$

The classes $\mathcal{N}_{\Sigma}(\alpha, \gamma)$ and $\mathcal{N}_{\Sigma}(\beta, \gamma)$ are defined in the following way :

Definition 3 . A function $f(z) \in \sum$ given by (1) is said to be in the class $\mathcal{N}_{\Sigma}$ if the following conditions are satisfied :

$\left|\arg \left(\frac{(1+\gamma) z^{2} f^{\prime \prime}(z)+z f^{\prime}(z)}{(1+\gamma) z f^{\prime}(z)-\gamma f(z)}\right)\right|<\frac{\alpha \pi}{2}(z \in U)(46)$

And

$\left|\arg \left(\frac{(1+\gamma) w^{2} g^{\prime \prime}(w)+w g^{\prime}(w)}{(1+\gamma) w g^{\prime}(w)-\gamma g(w)}\right)\right|<\frac{\alpha \pi}{2}(w \in U)(47)$

$(0<\alpha \leq 1 ; 0 \leq \gamma<1)$,

where the function $g=f^{-1}$ is given by (2) .

Definition 4 . A function $f(z) \in \sum$ given by (1) is said to be in the class $\mathcal{N}_{\Sigma}(\beta, \gamma)$ if the following conditions are satisfied :

$\operatorname{Re}\left(\frac{(1+\gamma) z^{2} f^{\prime \prime}(z)+z f^{\prime}(z)}{(1+\gamma) z f^{\prime}(z)-\gamma f(z)}\right)>\beta \quad(z \in U)$

And

$\operatorname{Re}\left(\frac{(1+\gamma) w^{2} g^{\prime \prime}(w)+w g^{\prime}(w)}{(1+\gamma) w g^{\prime}(w)-\gamma g(w)}\right)>\beta \quad(w \in U)$

$$
(0 \leq \beta<1 ; 0 \leq \gamma<1),
$$

where the function $g=f^{-1}$ is given by (2).

If we set $\gamma=0$ and $\tau=1$ in Theorem2. 1 and Theorem 3.1, then the classes $\mathcal{N}_{\Sigma m}(\tau, \gamma ; \alpha)$ and $\mathcal{N}_{\Sigma m}(\tau, \gamma ; \beta)$ reduce to the classes $\mathcal{N}_{\Sigma m}^{\alpha}$ and $S_{\Sigma m}^{\beta}$ investigated recently by Srivastava et al. [11] and thus, we obtain the following corollaries:

Corollary 6. Let $f(z) \in \mathcal{N}_{\Sigma m}^{\alpha}(0<\alpha \leq 1)$ be of the form (3) .Then

$\left|a_{m+1}\right| \leq \frac{2 \alpha}{\sqrt{\left[m(2 m+1)(m+1)-m(m+1)^{2}+m^{2}(m+1)^{2}(\alpha-1)\right]}}$

and

$\left|a_{2 m+1}\right| \leq \frac{\alpha}{m(2 m+1)}+\frac{2 \alpha(m+1)}{m^{3}(m+1)^{2}}$.

Corollary 7 . Let $f(z) \in \mathcal{N}_{\Sigma m}^{\alpha}(0 \leq \beta \leq 1)$ be of the form (4) . Then

$\left|a_{m+1}\right| \leq \sqrt{\frac{2(1-\beta)}{\left[m(2 m+1)(m+1)-m(m+1)^{2}\right]}}(52)$ and

$\left|a_{2 m+1}\right| \leq \frac{(1-\beta)}{m(2 m+1)}+\frac{2(1-\beta)^{2}(m+1)}{m^{3}(m+1)^{2}}(53)$

The classes $\mathcal{N}_{\Sigma m}^{\alpha}$ and $\mathcal{N}_{\Sigma m}^{\beta}$ are respectively defined as follows :

Definition 5 . A function $f(z) \in \sum_{m}$ given by (3) is said to be in the class $\mathcal{N}_{\sum_{m}}^{\alpha}$ if the following conditions are satisfied :

$\left|\arg \left\{\frac{z^{2} f^{\prime \prime}(z)}{z f^{\prime}(z)}+1\right\}\right|<\frac{\alpha \pi}{2}(z \in U)(54)$

and

$\left|\arg \left\{\frac{w^{2} g^{\prime \prime}(w)}{w g^{\prime}(w)}+1\right\}\right|<\frac{\alpha \pi}{2} \quad, \quad(w \in U)(55)$

and where the function $\mathrm{g}$ is given by (4) .

Definition 6. A function $f(z) \in \sum_{m}$ given by (3) is said to be in the class $\mathcal{N}_{\Sigma m}^{\beta}$ if the following

conditions are satisfied :

$\operatorname{Re}\left\{\frac{z^{2} f^{\prime \prime}(z)}{z f^{\prime}(z)}+1\right\}>\beta \quad(z \in U)(56)$

and

$\operatorname{Re}\left\{\frac{w^{2} g^{\prime \prime}(w)}{w g^{\prime}(w)}+1\right\}>\beta(w \in U)$.

$(0 \leq \beta<1)$

And where the function $\mathrm{g}$ is given by (4) .

\section{References}

[1] S. Altinkaya and S. yalcin , coefficient for certain subclasses of $\mathrm{m}$-fold symmetric bi-univalent function, Journal of Mathematics , Art . ID 241683, (2015) .

[2] S. Altinkaya and S. yalcin , on some subclasses of m-fold symmetric bi-univalent functions, Commun . Fac .Sci . univ.Ank. Series Al, 67(1) (2018).

[3] W. G. Atshan and N. A. J. Al-Ziadi, Coefficients bounds for a general subclasses of $\mathrm{m}$-fold symmetric bi-univalent functions, Journal of AL-Qadisiyah for Computer Science and Mathematics,9(2)(2017, 33-39.

[4] W. G. Atshan and N. A. J. Al-Ziadi,Coefficient Estimates of Bi-Univalent Functions Based on Subordination Involving Srivastava-Attiya Operator, International Journal of Advanced Research in Science, Engineering and Technology,4(9)(2017), 4574-4582.

[5] W. G. Atshan and R. A. Hiress, Coefficient estimates for subclasses of bi-univalent functions, Journal of AL-Qadisiyah for Computer Science and Mathematics, 10(3)(2018), 20-26. 
[6] P.L. Duren , univalent Functions , Grundlehren der Mathematischen Wissenschaften, Band 259, Springer Verlag , New York, Berlin, Heidelberg and Tokyo, 1983.

[7] S.S. Eker . coefficient bounds for subclasses of m-fold symmetric bi-univalent functions, Turk . J .Math., 40(2016).

[8] B.A. Frasin, Coefficient bounds for certain classes of biunivalent functions, Hacet .J .Math Stat . 43 (3)(2014).

[9] W. Koepf ,Coefficients of symmetric functions of bounded boundary rotations, proc . Amer. Math . Soc ., 105 (1989),324-329.

[10] C. Pommerenke, On the coefficients of close-to-convex functions, Michigan Math . J. g(1962), 259-269.

[11] H.M. Srivastava and D.Bansal, Coefficient estimates for asubclass of analytic and bi-univalent functions , J .Egypt . Math . Soc , 23 (2015), 242-246.

[12] H. M. Srivastava , S .Gaboury and F. G hanim , Coefficient estimates for some sub-classes of $\mathrm{m}$-fold symmetric bi-univalent functions, A ctauniversitatis A pulensis , 41 (2015) , 153-164.

[13] H.M. Srivastava , S.Gaboury and F.G hanim , Initial coefficient estimates for some subclasses of m-fold symmetric bi-univalent functions, A cta Mathematica Scientia , 36 B(3)(2016), 863-871.

[14] H.M. Srivastava, S .Sivasubramanian and R.Sivakumar, Initial coefficient bounds for a subclass of $\mathrm{m}$-fold symmetric bi-univalent functions, Tbilisi Math .J .7(2)(2014),1-10 .

[15] H. Tang , H.M. Srivastava, S . Sivasubramanian and P. Gurusamy, the Feketeszegö functional problems for some subclasses of m-fold symmetric bi-univalent functions ,J .Math .Ineq., 10(2016), 1063-1092 . 
مخمنات المعامل لبعض الاصناف الجزئية للاوال ثثائية التكافؤ المرتبطة بالطوية -m التناظرية

\author{
وقاص غالب عطشان 1 و \\ قسم الرياضيات / كليه علوم الحاسوب وتكنولوجيا المعلومات ق قلمب الرياضيات / كليه علوم الحاسوب / كلية علوم الحاسوب \\ وتكنولوجيا المعلومات \\ جامعة القادسيةـ الديوانية_العراق \\ جامعة القادسيةـ الايوانية_العراق
}

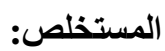

m- الغرض من البحث الحالي هو ان نقدم ونتحرى عن صنفين جزئيين جديدين و التحليلية في قرص الوحدة المفتوح ومن بين النتائج الاخرى للهذه الاصناف الجزئية حذود المعاملات العليا ( 